\title{
THE INFLUENCE OF THE PIEZOELECTRIC EFFECT ON THE ANALYSIS OF ELASTIC STRESSES FOR A COMPOSITE DISK UNDER THE PARABOLIC DISTRIBUTION OF TEMPERATURE
}

\author{
BERRABAH HAMZA MADJID* \\ Laboratoire Matériaux et Hydrologie, Centre Universitaire Ahmed Zabana \\ Relizane, Relizane, 48000, Algérie
}

[Received 17.09.2018. Accepted 18 February 2019]

doi: 10.7546/JTAM.49.19.01.02

\begin{abstract}
In this paper, we studied the influence of the piezoelectric effect under the variation of the parabolic temperature, for this purpose, a thermoelastic composite disk reinforced by steel fibers is used for the analysis of the radial and tangential elastic thermal stresses. The solutions of the stresses were obtained analytically. In addition, a comparison between these expressions for different temperature values is calculated.
\end{abstract}

KEY WORDS: piezoelectric, radial, temperature, tangential, elastic.

\section{INTRODUCTION}

Thermoplastic composites offer many advantages in industrial applications due to such as high-improved fracture toughness, specific stiffness, and increased impact resistance. They can be remelted, reformed and reformed. Thermal stress analysis in composite discs is a considerable subject due to the wide range of applications in practice [1]. The fiber reinforced composite materials are widely used in many structural applications such as marine, automotive and aviation industries, on account of their high strength, low weight, good fatigue life and corrosion resistance [2]. Piezoelectric materials have electro-mechanical coupling characteristics, and have been widely used in sensors and actuators $[3,4]$.

Materials capable of tolerating material properties with spatial dimensions offer reliable engineering solutions for industrial applications involving severe thermal and mechanical loading as in the case of tribology, combustion processes, aerospace structures and high temperature technologies [5].

An analytical method to investigate thermo-elastic stress analysis on an orthotropic composite disc with aluminium metal-matrix reinforced towards $x$ - and $y$-direction has been used in Ref. [6]. The elastic stress analysis of the hollow composite disc under the linearly increasing distribution of temperature has been carried out analytically with the help of a computer program [7].

\footnotetext{
${ }^{*}$ Corresponding author e-mail: b_hamza_2005@yahoo.fr
} 
The experimental and theoretical study of dielectric, piezoelectric and mechanical properties of these materials is performed in our laboratories, using various methods, including interferometric method, operating in a wide temperature range [8].

The variation of the elastic thermal stresses with the presence of the piezoelectric effect was analysed under a parabolic temperature loading for different values of $(r)$ for a thermoplastic composite disc.

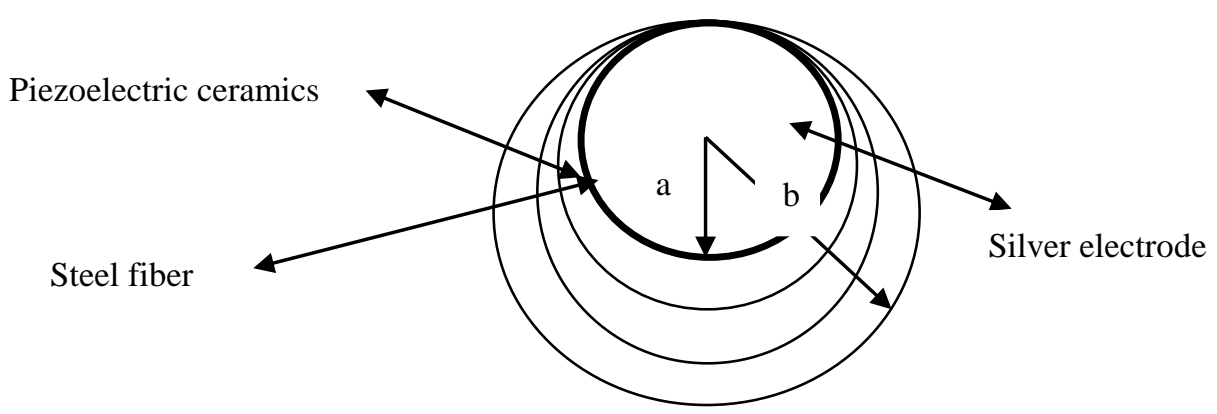

Fig. 1. Structure of the piezoelectric composite disc.

The stress-strain relationship for a piezoelectric composite disk under the variation of a parabolic temperature is expressed as follows:

$$
\begin{aligned}
\varepsilon_{r} & =\frac{1}{E_{r}} \sigma_{r}-\frac{v_{r \theta}}{E_{\theta}} \sigma_{\theta}+\alpha_{r} T, \\
\varepsilon_{\theta} & =\frac{v_{r \theta}}{E_{\theta}} \sigma_{r}-\frac{1}{E_{\theta}} \sigma_{\theta}+\alpha_{\theta} T,
\end{aligned}
$$

where

$$
\begin{aligned}
& \sigma_{r}-\text { the radial stress; } \\
& \sigma_{\theta}-\text { the tangential stress; } \\
& \varepsilon_{r} \text { - the radial strain; } \\
& \varepsilon_{\theta}-\text { the tangential strain; } \\
& \alpha_{r}-\text { the thermal expansion coefficients in the radial direction; } \\
& \alpha_{\theta}-\text { the thermal expansion coefficients in the tangential direction; } \\
& E_{r}-\text { modulus of elasticity in the radial direction; } \\
& E_{\theta}-\text { modulus of elasticity in the tangential direction. }
\end{aligned}
$$


The equation of equilibrium and the relation between deformations is written as follows:

$$
\begin{gathered}
r \frac{\partial \sigma_{r}}{\partial r}+\sigma_{r}-\sigma_{\theta}=0, \\
\varepsilon_{r}=\varepsilon_{\theta}+r \frac{\partial \varepsilon_{r}}{\partial r}+d_{31} E_{3} .
\end{gathered}
$$

For the two stresses a certain function is used to determine the differential equation:

$$
\begin{aligned}
\sigma_{r} & =\frac{F}{r}, \\
\sigma_{\theta} & =\frac{\partial F}{\partial r} .
\end{aligned}
$$

Using equations (1), (2), (5), and (6) and substituting in (4) we obtain

(7) $\frac{1}{E_{r}} \frac{F}{r}+\frac{v_{r \theta}}{E_{\theta}} \frac{\partial F}{\partial r}+\alpha_{r} T=\frac{v_{r \theta}}{E_{\theta}} \frac{F}{r}+\frac{1}{E_{\theta}} \frac{\partial F}{\partial r}$

$$
+\alpha_{\theta} T+\frac{v_{r \theta}}{E_{\theta}} \frac{\partial F}{\partial r}+\frac{r}{E_{\theta}} \frac{\partial^{2} F}{\partial r^{2}}+r \alpha_{\theta} \frac{\partial T}{\partial r}+d_{31} E_{3} .
$$

Another way,

(8) $\frac{r^{2}}{E_{\theta}} \frac{\partial^{2} F}{\partial r^{2}}+\frac{r}{E_{\theta}} \frac{\partial F}{\partial r}+\left(\frac{v_{r \theta}}{E_{\theta}}-\frac{1}{E_{r}}\right) F=-r^{2} \alpha_{\theta} \frac{\partial T}{\partial r}+\left(r \alpha_{r}-r \alpha_{\theta}\right) T-d_{31} E_{3}$.

In this study the formula of parabolic variation of temperature is given by

$$
T=\frac{T_{i}}{\left(b^{2}-a^{2}\right)}\left(b^{2}-r^{2}\right) .
$$

The differential equation of function $F$ is written as follows:

(10) $\frac{r^{2}}{E_{\theta}} \frac{\partial^{2} F}{\partial r^{2}}+\frac{r}{E_{\theta}} \frac{\partial F}{\partial r}+\left(\frac{v_{r \theta}}{E_{\theta}}-\frac{1}{E_{r}}\right) F$

$$
=\left(-2 r^{3} \frac{\alpha_{\theta}}{\left(b^{2}-a^{2}\right)}-\frac{\left(r \alpha_{r}-r \alpha_{\theta}\right)\left(b^{2}-r^{2}\right)}{\left(b^{2}-a^{2}\right)}\right) T+d_{31} E_{3} .
$$

Another way to reduce this equation

$$
r^{2} \frac{\partial^{2} F}{\partial r^{2}}+r \frac{\partial F}{\partial r}+\left(v_{r \theta}-\frac{E_{\theta}}{E_{r}}\right) F+L_{1} T+E_{\theta} d_{31} E_{3}
$$


where

$$
\begin{gathered}
L_{1}=\left(-3 \frac{E_{\theta} \alpha_{\theta}}{\left(b^{2}-a^{2}\right)}+\frac{E_{\theta} \alpha_{r}}{\left(b^{2}-a^{2}\right)}\right) r^{3}+\left(\frac{E_{\theta} \alpha_{r} b^{2}}{\left(b^{2}-a^{2}\right)}+\frac{E_{\theta} \alpha_{\theta} b^{2}}{\left(b^{2}-a^{2}\right)}\right) r, \\
r^{2} \frac{\partial^{2} F}{\partial r^{2}}+r \frac{\partial F}{\partial r}+\left(v_{r \theta}-\frac{E_{\theta}}{E_{r}}\right) F+L_{2},
\end{gathered}
$$

where

$$
L_{2}=L_{1} T+E_{\theta} d_{31} E_{3} .
$$

The solution of the differential equation gives

$$
F=-\frac{L_{2} E_{r}}{v_{r \theta} E_{r}-E_{\theta}}+C_{1} r \frac{\sqrt{-E_{r}\left(v_{r \theta} E_{r}-E_{\theta}\right)}}{E_{r}}+C_{2} r \frac{-\sqrt{-E_{r}\left(v_{r \theta} E_{r}-E_{\theta}\right)}}{E_{r}},
$$

where $C_{1}, C_{2}$ are the constants of integrations,

$$
\begin{aligned}
P & =-\frac{L_{2} E_{r}}{v_{r \theta} E_{r}-E_{\theta}}, \\
G & =\frac{\sqrt{-E_{r}\left(v_{r \theta} E_{r}-E_{\theta}\right)}}{E_{r}} .
\end{aligned}
$$

With a simpler expression $F$ is written as follows:

$$
F=P+C_{1} r^{G}+C_{2} r^{-G} .
$$

We substitute the expression of $F$ in the two formulas of the radial and tangential stresses:

$$
\begin{aligned}
\sigma_{r} & =\frac{F}{r}=\frac{P}{r}+C_{1} r^{G-1}+C_{2} r^{-G-1}, \\
\sigma_{\theta} & =\frac{\partial F}{\partial r}=P_{1}+C_{1} G r^{G-1}-C_{2} G r^{-G-1},
\end{aligned}
$$

where

(21) $P_{1}=\frac{\partial P}{\partial r}=3\left(-3 \frac{E_{\theta} \alpha_{\theta}}{\left(b^{2}-a^{2}\right)}+\frac{E_{\theta} \alpha_{r}}{\left(b^{2}-a^{2}\right)}\right) r^{3}+\left(\frac{E_{\theta} \alpha_{r} b^{2}}{\left(b^{2}-a^{2}\right)}+\frac{E_{\theta} \alpha_{\theta} b^{2}}{\left(b^{2}-a^{2}\right)}\right)$,

(22) $\sigma_{r}=0$ at $r=a$ and $r=b$.

The expressions of $C_{1}$ and $C_{2}$ are given by

$$
\begin{aligned}
& C_{1}=-\frac{P\left(b^{-G}-a^{-G}\right)}{\left(a^{G} b^{-G}-b^{G} a^{-G}\right)}, \\
& C_{2}=-\frac{P\left(a^{G}-b^{G}\right)}{\left(a^{G} b^{-G}-b^{G} a^{-G}\right)} .
\end{aligned}
$$




\section{RESUlTS AND Discussion}

$E_{\theta}=11300 \mathrm{Mpa}, E_{r}=260 \mathrm{Mpa}, v_{r \theta}=0.43, \alpha_{r}=130 \times 10^{-6} 1 /{ }^{\circ} \mathrm{C}, \alpha_{\theta}=12.8 \times$ $10^{-6} 1 /{ }^{\circ} \mathrm{C}, a=30 \mathrm{~mm}, b=70 \mathrm{~mm}, d_{31}=-5.35 \times 10^{-7} \mathrm{~mm} / \mathrm{v}, E_{3}=7150 \mathrm{v} / \mathrm{mm}$.

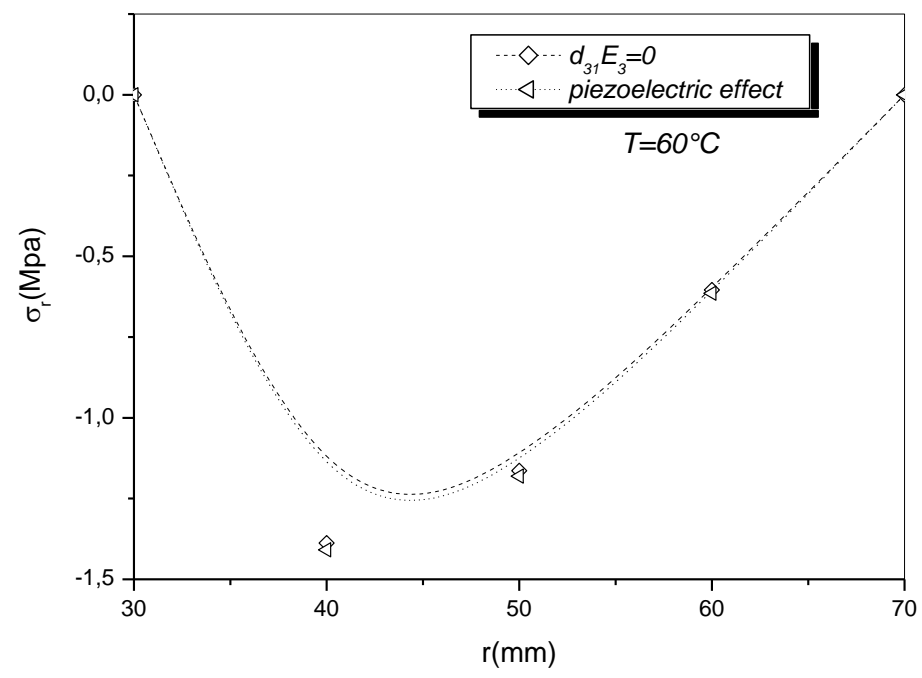

Fig. 2. Variation of the radial stress at $60^{\circ} \mathrm{C}$.

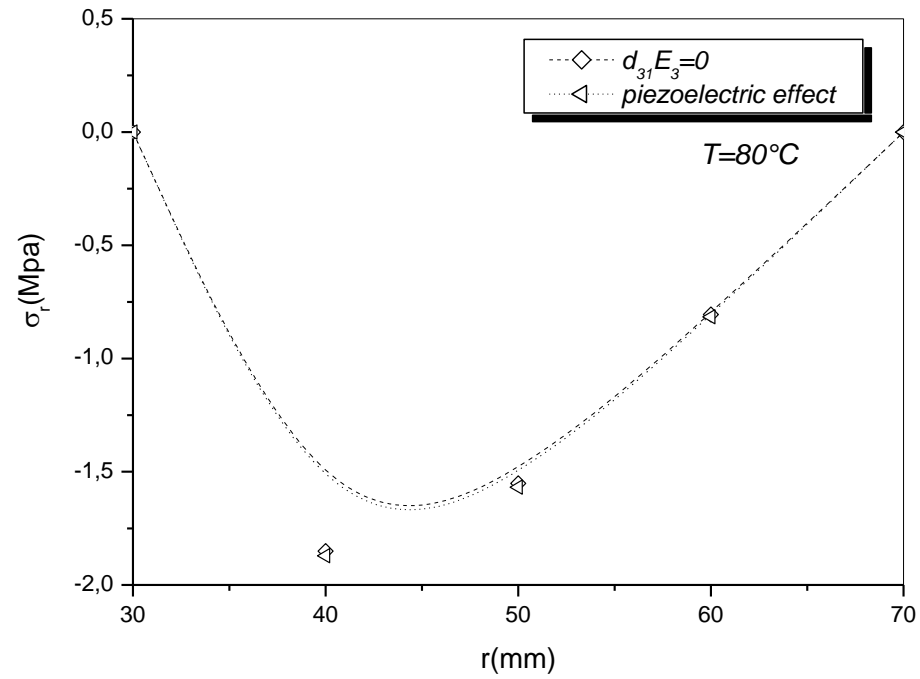

Fig. 3. Variation of the radial stress at $80^{\circ} \mathrm{C}$.

The variation of the thermo-elastic radial stresses for the three different temperature values $60^{\circ} \mathrm{C}, 80^{\circ} \mathrm{C}$ and $100^{\circ} \mathrm{C}$ with the presence of the piezoelectric effect are 
The Influence of the Piezoelectric Effect on the Analysis of Elastic Stresses...

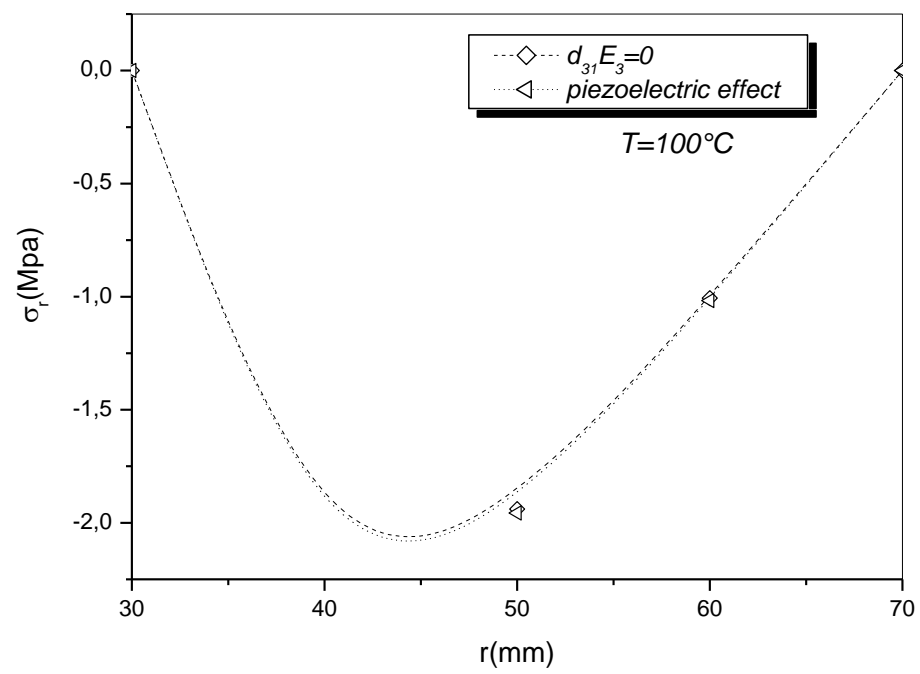

Fig. 4. Variation of the radial stress at $100^{\circ} \mathrm{C}$.

shown in Figs. 2, 3 and 4, respectively. These stresses augmented with small ratios include this effect; these figures show that all the values of these stresses give the form of compression between the two ends, inner and outer diameter.

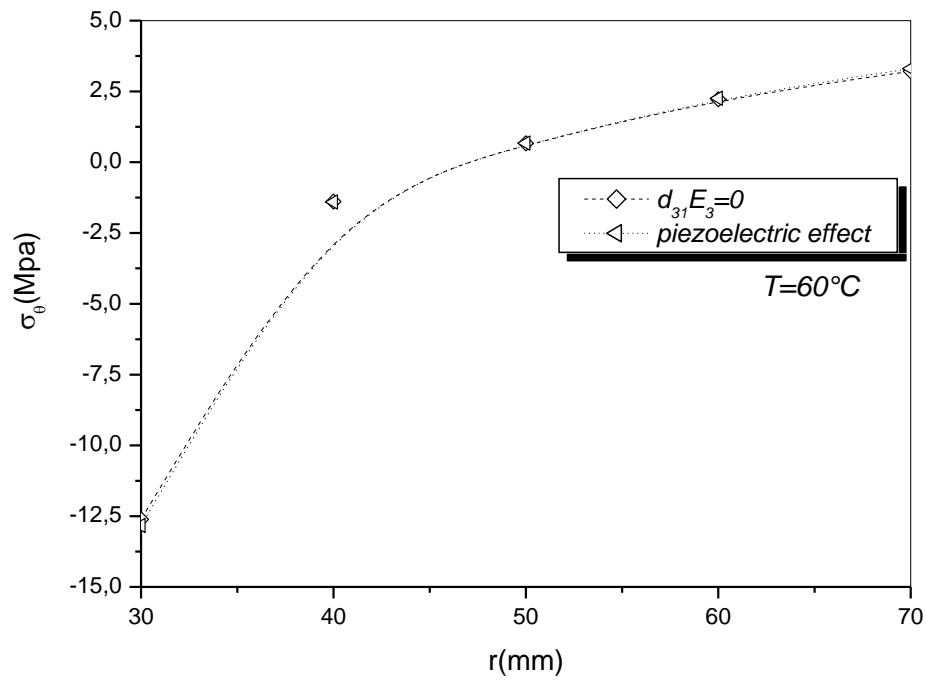

Fig. 5. Variation of the tangential stress at $60^{\circ} \mathrm{C}$. 


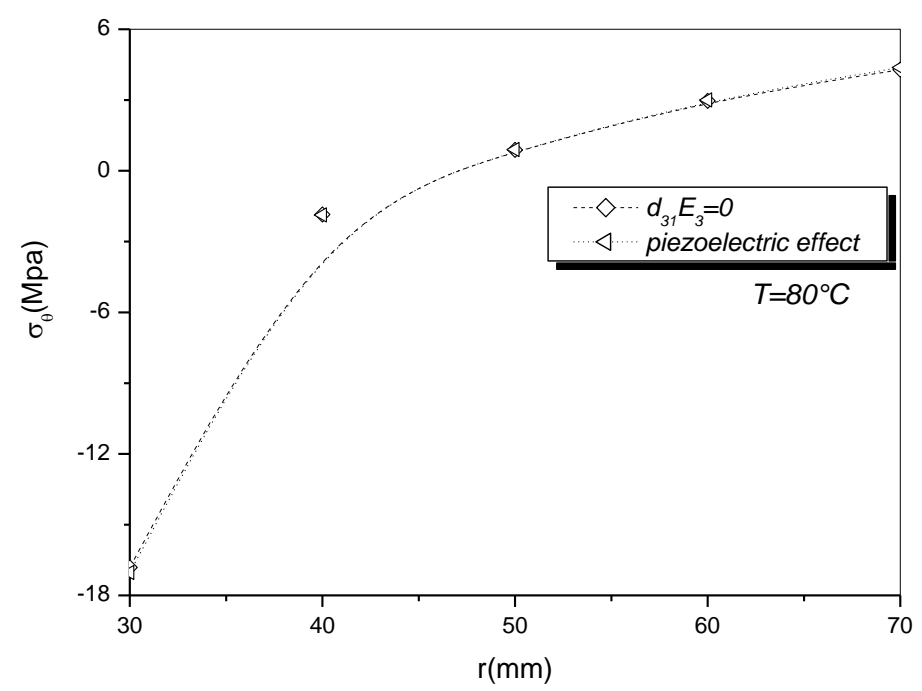

Fig. 6. Variation of the tangential stress at $80^{\circ} \mathrm{C}$.

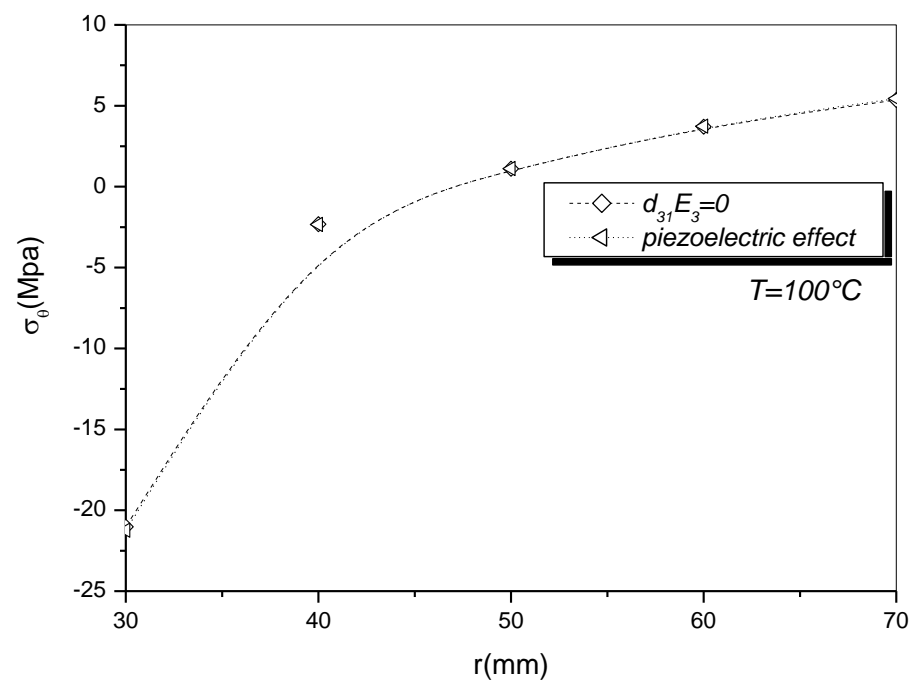

Fig. 7. Variation of the tangential stress at $100^{\circ} \mathrm{C}$.

Figures 5, 6 and 7 show the tangential stress variation for $T=60^{\circ} \mathrm{C}, 80^{\circ} \mathrm{C}$ and $100^{\circ} \mathrm{C}$, the influence of the piezoelectric effect is clear in this part, in this part the two phenomena s' is produced, compression and traction, especially in areas where $a=30 \mathrm{~mm}$ and $b=70 \mathrm{~mm}$, the figures show that the tangential stresses at the inner diameter are higher than at the outer diameter. 


\section{CONCLUSion}

In this study, the variation of the radial and tangential thermo-elastic stresses for a composite disk reinforced by steel fibers is of such great importance that the different values obtained along the diameter, the piezoelectric effect positively affects two types of stresses, a convergence of all the values at zero at the level $r=a$ and $r=b$, the compression and tensile phenomena is illustrated in all the graphs, the radial stresses reaches its maximum in the middle between $a$ and $b$ which causes the risk that this disc will be spread.

\section{REFERENCES}

[1] F. Sen, Y. Pekbey, O. Sayman (2007) Elastic-Plastic Stress Analysis of a Thermoplastic Composite Disc under Parabolic Temperature Distribution. Indian Journal of Engineering and Materials Sciences. 14 (2007) 282-288.

[2] F. SEN, M. SAYER (2010) Influences of Multiple Holes on Thermal Stresses in a Thermoplastic Composite Disc. Indian Journal of Engineering and Materials Sciences 17 157-168.

[3] X.D. Sun, W.Z. Yuan, D.Y. QiaO, S. Ren (2017) The Analysis of the SelfOscillation System for Resonant Pressure Sensor. Microsystem Technology 23 945-951.

[4] K.T. Chang, C.W. Lee (2008) Fabrication and Characteristics of Thin Disc Piezoelectric Transformers Based on Piezoelectric Buzzers with Gap Circles. Ultrasonics 48 91-97.

[5] C. EvcI, M. GÜLGEÇ (2018) Functionally Graded Hollow Cylinder under Pressure and Thermal Loading: Effect of Material Parameters on Stress and Temperature Distributions. International Journal of Engineering Science 123 92-108.

[6] O. SAYMAn (2004) Thermal Stress Analysis in an Aluminum Metal-Matrix Disc. Journal of Reinforced Plastics and Composites 23 1473-1479.

[7] G. Altan (2004) Thermal Stress Analysis of Composite Discs. MSc Thesis, Pamukkale University, Denizli, Turkey.

[8] L. Burianova, A. Kopal, J. Nosek (2003) Characterization of Advanced Piezoelectric Materials in the Wide Temperature Range. Materials Science and Engineering B99 187-191. 